\title{
Real sector of the nonminimally coupled scalar field to self-dual gravity
}

\author{
Merced Montesinos ${ }^{a, b *}$, Hugo A. Morales-Técotl ${ }^{c \dagger}$, \\ Luis F. Urrutia ${ }^{d \ddagger}$ and J. David Vergara ${ }^{d \S}$ \\ ${ }^{a}$ Department of Physics and Astronomy, University of Pittsburgh, \\ Pittsburgh, PA 15260, USA. \\ ${ }^{b}$ Departamento de Física, Centro de Investigación y de Estudios Avanzados del I.P.N., \\ Av. I.P.N. No. 2508, 07000 México D.F., México. \\ ${ }^{c}$ Departamento de Física, Universidad Autónoma Metropolitana-Iztapalapa, \\ Apartado Postal 55-534, 09340, México D.F., México. \\ ${ }^{d}$ Instituto de Ciencias Nucleares, Universidad Nacional Autónoma de México, \\ Apartado Postal 70-543, 04510, México D.F., México.
}

\begin{abstract}
A scalar field non-minimally coupled to gravity is studied in the canonical framework, using self-dual variables. The corresponding constraints are first class and polynomial. To identify the real sector of the theory, reality conditions are implemented as second class constraints, leading to three real configurational degrees of freedom per space point. Nevertheless, this realization makes non-polynomial some of the constraints. The original complex symplectic structure reduces to the expected real one, by using the appropriate Dirac brackets. For the sake of preserving the simplicity of the constraints, an alternative method preventing the use of Dirac brackets, is discussed. It consists of converting all second class constraints into first class by adding extra variables. This strategy is implemented for the pure gravity case.
\end{abstract}

PACS: 04.20.Fy, 04.60.Ds

Typeset using REVTEX

\footnotetext{
*E-mail: merced@fis.cinvestav.mx

$\dagger$ Associate member of Abdus Salam-ICTP, Trieste, Italy. E-mail: hugo@xanum.uam.mx

${ }^{\ddagger}$ E-mail: me@aurora.nuclecu.unam.mx

§E-mail: vergara@nuclecu.unam.mx
} 


\section{INTRODUCTION}

The failure of using perturbation theory to quantize General Relativity (GR) has led to different approaches, like string theory and canonical quantization, which intend to define quantum gravity [1]. Instead of incorporating the remaining fundamental interactions as the former, the latter approach consists of just adopting standard quantum theory and classical GR. The spacetime structure, on the other hand, seems to be sensible to the non-perturbative aspects modeling it [2].3]. This is in itself a major motivation for studying canonical GR. Some success has been achieved within canonical quantum gravity since the late eighties, after the introduction, by Ashtekar [4], of a set of complex canonical variables which greatly simplify the form of the constraints to which the theory is reduced. The kernel of this algebra of constraints defines the space of physical states in the theory and some formal elements of it have been constructed [1.

In spite of its simplicity, the constraints of GR in terms of Ashtekar variables describe complex gravity. Ashtekar himself considered reducing to the real sector, through the introduction of an inner product designed to make hermitian the physical operators. However, this strategy has not worked up to now, except for some particular cases. Other alternatives have been presented to avoid the use of reality conditions, at the price of a more cumbersome form of the constraints. Yet, quantum mechanically, the situation seems tractable [3].

There still remains the possibility of keeping the self-dual canonical formalism and trying to envisage how to select the real sector of the theory. Indeed this is possible, as it has been shown for pure gravity in [5], at the classical level. Reality conditions are implemented as second class constraints. The present work is devoted to show how this result is also valid in the case of a scalar field non-minimally coupled to gravity.

The strategy followed in this paper is an extension of the pure gravity analysis of [5], to incorporate a non-minimally coupled scalar field . It consists of five steps: (i) each complex canonical variable is splitted into one real and one imaginary degrees of freedom; (ii) then, every real and imaginary part is regarded as a configuration variable. An extended phase 
space is hence defined, where the corresponding momenta will arise directly from the action. Notice that the definition of such momenta will produce primary constraints; (iii) next, the reality conditions are implemented upon the splitting, as additional primary constraints. The criterion being to restrict to a real three-metric together with a real scalar field, during the whole evolution of the system; (iv) after imposing the conservation of the constraints, which amounts to obtaining all possible secondary constraints, a final classification of the full set into first and second class is performed. This may require the redefinition of some of them; and finally, (v) the issue of how to deal with the resulting set of second class constraints should be addressed. Since the use of Dirac brackets take the first class constraints back to its Palatini canonical form, which is highly non-polynomial, some alternatives should be tried.

A review of how to select the real sector in phase space of pure self-dual gravity is made in section II. It includes the result of converting all second class into first class constraints, by introducing extra canonical variables. Thus, Dirac brackets are not used. Next, the extension of the method to the case of the scalar field non-minimally coupled to gravity is described in section III. The polynomial form of the constraints for the complex theory is exhibited, as well as the whole set of first and second class constraints describing the real sector of the theory. Finally, the last section contains some conclusions and perspectives. A possibility regarding how to avoid the use of Dirac brackets to eliminate the second class constraints for the scalar field case is briefly discussed. A point on notation: $\tilde{E}^{a i}$ is considered here as a complex density inverse triad of weight one, whilst $\tilde{e}^{a i}$ is its real part. This is just the opposite convention of ref. [5], but it has the advantage that adjusts to the rest of the literature [4]. The number of over and under tildes represent the positive or negative density weight $( \pm 1, \pm 2$, etc.) respectively, unless it is obvious from the definition of the different variables. In the case of the $\delta(\tilde{x}, y)$ the tilde indicates it is a density of weight one in the argument $x$. 


\section{PURE GRAVITY}

The Ashtekar complex canonical variables are: (i) $\tilde{E}^{a i}:=E E^{a i}$, with $E^{a i}$ being the triad $\left(E^{a i} E_{i}^{b}:=q^{a b}, q^{a b}\right.$ is the spatial three-metric), and $a, b, \ldots=1,2,3$ are spatial indices, whereas $i, j, \ldots=1,2,3$ are $\mathrm{SO}(3)$ internal indices. Also, $E:=\operatorname{det} E_{b j}$ with $E_{b j}$ being the inverse of $E^{a i}$. (ii) $A_{a i}$ is the three-dimensional projection of the self-dual connection [4], with associated covariant derivative $\mathcal{D}_{a} \lambda_{i}=\partial_{a} \lambda_{i}+\epsilon_{i j k} A_{a}{ }^{j} \lambda^{k}$ and $F_{a b}{ }^{i}:=\partial_{a} A_{b}{ }^{i}-\partial_{b} A_{a}{ }^{i}+\epsilon^{i}{ }_{j k} A_{a}{ }^{j} A_{b}{ }^{k}$ is the corresponding curvature. In terms of these variables, the self-dual action of canonical GR is given by

$$
S=\int d t d^{3} x\left\{-i \tilde{E}^{a i} \dot{A}_{a i}-N \mathcal{S}-N^{a} \mathcal{V}_{a}-N^{i} \mathcal{G}_{i}\right\}
$$

where

$$
\mathcal{S}:=\epsilon_{i j k} \tilde{E}^{a i} \tilde{E}^{b j} F_{a b}{ }^{k}, \quad \mathcal{V}_{a}:=\tilde{E}^{b}{ }_{j} F_{a b}{ }^{j}, \quad \mathcal{G}_{i}:=\mathcal{D}_{a} \tilde{E}^{a}{ }_{i},
$$

are the constraints of the theory and $N, N^{a}, N^{i}$ are Lagrange multipliers. Such constraints are first class and polynomial in the phase space variables. Let us denote by $\mathcal{R}$ any of them and by $\{\mathcal{R}\}$ the full set.

Notice that having 18 complex phase space variables $\left(A_{a i}, \tilde{E}^{b j}\right)$, together with 7 complex first class constraints, $\{\mathcal{R}\}$, leaves us with 2 complex configurational degrees of freedom. Then, in order to recover the 2 real configurational degrees of freedom per point, further constraints are necessary. To this end, let us introduce the splitting

$$
\tilde{E}^{a i}=\tilde{e}^{a i}+i \tilde{\mathcal{E}}^{a i}, \quad A_{b j}=\gamma_{b j}-i K_{b j}
$$

From now on, all the above thirty six degrees of freedom are taken as configuration variables in the action (11). Hence, the associated canonical momenta $\Pi$ lead to the primary constraints $\Phi_{\mathcal{E} a i}=\Pi_{\mathcal{E} a i}, \quad \Phi_{\gamma}^{a i}=\Pi_{\gamma}^{a i}+i \tilde{e}^{a i}, \quad \Phi_{K}^{a i}=\Pi_{K}^{a i}+\tilde{e}^{a i}, \quad \Phi_{e a i}=\Pi_{e a i}$, which as a set is denoted by $\{\Phi\}$. The coordinates of the total phase space are $Y_{A}=$ $\left(\tilde{e}^{a i}, \tilde{\mathcal{E}}_{a i}, \gamma^{a i}, K_{a i}, \Pi_{e a i}, \Pi_{\mathcal{E} a i}, \Pi_{\gamma}^{a i}, \Pi_{K}^{a i}\right)$. 
The reduction of the complex phase space $\left(A_{a i}, \tilde{E}^{b j}\right)$ to a real one is achieved by means of the following reality conditions

$$
\psi^{a i}:=\tilde{\mathcal{E}}^{a i}=0, \quad \chi_{a i}:=\gamma_{a i}-f_{a i}(\tilde{e})=0,
$$

which are subsequently taken as additional primary constraints. The constraints $\psi^{a i}$ enforces the $\tilde{E}^{a i}$ to be real, and hence the corresponding three-metric. The constraints $\chi_{a i}$ ensures that, upon evolution, $\tilde{E}^{a i}$ keeps being real. Using the compatibility condition between a real torsion-free connection and the triad, the form of $f_{a i}$ is chosen as $f_{a i}=\frac{1}{2}\left[e_{a i} e_{\sim}{ }^{j} \epsilon_{j r s}-\right.$ $\left.2 e_{a j} e_{\sim}{ }^{j} \epsilon_{i r s}\right] \tilde{e}^{d r} \partial_{d} \tilde{e}^{c s}$. Let us observe that $\chi_{a i}$ is not polynomial in $\tilde{e}^{b j}$.

The full set of primary constraints is $\{\{\mathcal{R}\}, \psi, \chi,\{\Phi\}\}$, written in terms of the real variables $Y_{A}$. The evolution of the primary constraints does not introduce additional constraints. After redefining $\Phi_{e a i} \rightarrow \Phi^{\prime}{ }_{e a i}=\Phi_{e a i}+\alpha_{a i b j} \Phi_{\gamma}{ }^{b j}+\beta_{a i}{ }^{b j} \chi_{b j}+\eta_{a i b j} \Phi_{K}{ }^{b j}$, the Poisson brackets matrix for the subset $\{\Xi\}=\left\{\left\{\Phi^{\prime}\right\}, \psi, \chi\right\}$ reveals them as second class constraints. Besides having a simple form, it is a phase space independent, block diagonal matrix with non zero determinant.

To keep $\{\mathcal{R}\}$ as a first class set it is enough to redefine each element as $\mathcal{R}^{\prime}=\mathcal{R}+$ $\left\{\Phi_{\mathcal{E} b j}, \mathcal{R}\right\} \psi^{b j}+\left\{\Phi_{\gamma}^{b j}, \mathcal{R}\right\} \chi_{b j}+\left\{\Phi^{\prime}{ }_{e b j}, \mathcal{R}\right\} \Phi_{K}{ }^{b j}-\left\{\Phi_{K}{ }^{b j}, \mathcal{R}\right\} \Phi^{\prime}{ }_{e b j}$, so that they have zero Poisson brackets with the previous second class subset. This redefinition preserves the property $\left\{\mathcal{R}^{\prime}, \mathcal{Q}^{\prime}\right\} \approx 0$, for any pair of constraints in $\left\{\mathcal{R}^{\prime}\right\}$. In this way, there are no additional contributions to the set of primary constraints $\{\Upsilon\}:=\left\{\mathcal{R}^{\prime}\right\} \cup\{\Xi\}$, which includes the reality conditions. Counting the independent variables gives 2 real configurational degrees of freedom per space point, as it should be for real GR [5].

At this point Dirac's programme calls for the elimination of the second class constraints through the use of Dirac brackets. This, however, would yield a cumbersome form for the remaining constraints. One might avoid such treatment of the second class constraints by transforming them into first class constraints. To achieve this, by means of the BatalinTyutin procedure [0, [], one adds a new canonical pair, $\left\{Q^{a i}, P_{b j}\right\}=\delta_{a}{ }^{b} \delta_{i}{ }^{j} \delta^{(3)}$, per original couple of second class constraints, i.e., the phase space is further enlarged with the new 
variables $\Psi_{\Xi}=\left(Q_{\mathcal{E}}{ }^{a i}, Q_{\gamma a i}, Q_{e}{ }^{a i}, P_{\mathcal{E} a i}, P_{\gamma}{ }^{a i}, P_{e a i}\right)$. In the present case, the set of first class constraints replacing the former second class set is

$$
\begin{aligned}
\bar{\psi}^{a i} & :=\tilde{\mathcal{E}}^{a i}+Q_{\mathcal{E}}{ }^{a i}, \\
\bar{\Phi}_{\mathcal{E} a i} & :=\Pi_{\mathcal{E} a i}-P_{\mathcal{E} a i} \\
\bar{\chi}_{a i} & :=\gamma_{a i}-f_{a i}(\tilde{e})+Q_{\gamma a i}, \\
\bar{\Phi}_{\gamma}{ }^{a i} & :=\Pi_{\gamma}^{a i}+i \tilde{e}^{a i}-P_{\gamma}^{a i} \\
\bar{\Phi}_{K}{ }^{a i} & :=\Pi_{K}^{a i}+\tilde{e}^{a i}+Q_{e}^{a i} \\
\bar{\Phi}^{\prime}{ }_{e a i} & :=\Phi_{e a i}+\alpha_{a i b j} \Phi_{\gamma}^{b j}+\beta_{a i}^{b j} \chi_{b j}+\eta_{a i b j} \Phi_{K}^{b j}-P_{e a i},
\end{aligned}
$$

which reduces to the original set by setting $Q^{a i}=0=P_{b j}$. Let us denote any of the constraints in (5) by $\bar{\Xi}_{\Lambda}$. Any pair satisfies $\left\{\bar{\Xi}_{\Lambda}, \bar{\Xi}_{\Lambda^{\prime}}\right\}=0$; i.e. the set (5) is first class. Next, it is necessary to keep the set $\left\{\mathcal{R}^{\prime}\right\}$ first class. This can be done by recalling that the Poisson brackets matrix among the constraints $\{\Xi\}$ is independent of the phase space variables and by following the method of [7]. Thus, one redefines $\mathcal{R}^{\prime}$ as

$$
\overline{\mathcal{R}}^{\prime} \equiv \mathcal{R}^{\prime}(Y-\bar{Y})
$$

where

$$
\begin{aligned}
Y_{A}-\bar{Y}_{A}:= & \left\{\tilde{e}^{a i}-Q_{e}{ }^{a i}, \mathcal{E}^{a i}+Q_{\mathcal{E}}^{a i}, \gamma_{a i}+Q_{\gamma a i}+Q_{e}{ }^{b j} \frac{\delta f_{a i}}{\delta \tilde{e}^{b j}}, K_{a i}+P_{e a i}+i Q_{e}{ }^{b j} \frac{\delta f_{b j}}{\delta \tilde{e}^{a i}}\right. \\
& \Pi_{e a i}-P_{e a i}-i Q_{\gamma a i}+P_{\gamma}{ }_{\gamma} j \frac{\delta f_{b j}}{\delta \tilde{e}^{a i}}+Q_{e}{ }^{b j}\left(\frac{\delta^{2} f_{c k}}{\delta \tilde{e}^{a i} \delta \tilde{e}^{b j}} \Phi_{\gamma}^{c k}+i \frac{\delta^{2} f_{a i}}{\delta \tilde{e}^{c k} \delta \tilde{e}^{b j}} \Phi_{K}^{c k}+i \frac{\delta f_{b j}}{\delta \tilde{e}^{a i}}\right) \\
& \left.\Pi_{\mathcal{E} a i}-P_{\mathcal{E} a i}, \Pi_{\gamma}{ }^{a i}-P_{\gamma}{ }^{a i}-i Q_{e}{ }^{a i}, \Pi_{K}{ }^{a i}\right\}
\end{aligned}
$$

The set (6) is in involution with $\left\{\bar{\Xi}_{\Lambda}\right\}$, i.e. $\left\{\overline{\mathcal{R}}^{\prime}, \bar{\Xi}_{\Lambda}\right\}=0$. Hence, the final whole set of constraints is first class and contains an Abelian ideal: $\{\bar{\Xi}\}$. The non-Abelian sector is just given by $\left\{\overline{\mathcal{R}}^{\prime}\right\}$. By construction, this sector preserves the structure of the first class algebra among the elements of $\left\{\mathcal{R}^{\prime}\right\}$. Notice that the set $\{\mathcal{R}\}$ depends only on the configurational variables $(\tilde{e}, \mathcal{E}, \gamma, K)$. In this way, the most involved modifications to $\left\{\mathcal{R}^{\prime}\right\}$, via Eq.(可), come from the terms that are proportional to the second class constraints. It is worth 
emphasizing that all the non-polynomiality of the constraints $\left\{\left\{\overline{\mathcal{R}}^{\prime}\right\} \cup\{\bar{\Xi}\}\right\}$ arises only through one function, which is $f_{a i}(\tilde{e})$, appearing in the reality conditions (雨). Thus, one might think that a choice of (41) in a polynomial form would solve this undesirable feature. However, as shown in [5], this not the case and one should look for alternative approaches.

\section{NON-MINIMAL SELF-DUAL ECKG THEORY}

The second order action with scalar field non-minimally coupled to gravity is given by

$$
S\left[g^{a b}, \phi\right]=\int_{M} d^{4} x\left\{\sqrt{-g} \mathcal{R}-\frac{1}{2} \sqrt{-g}\left(g^{a b} \partial_{a} \phi \partial_{b} \phi+\left(m^{2}+\xi \mathcal{R}\right) \phi^{2}\right)\right\},
$$

where $\xi$ is a dimensionless constant. The canonical analysis of this action has been developed in 8 . In a first order formalism one can adopt instead

$$
\begin{aligned}
S\left[\omega^{\prime}, e, \phi\right] & =\int_{M} d^{4} x\left\{\frac{1}{2} e e_{I}^{a} e_{J}^{b} \Omega^{2} R_{a b}^{\prime}{ }^{I J}\left[\omega^{\prime}\right]-\frac{1}{2} e\left(e_{I}^{a} e^{b I} \partial_{a} \phi \partial_{b} \phi+m^{2} \phi^{2}\right)\right\}, \\
\Omega^{2} & :=1-\xi \phi^{2} .
\end{aligned}
$$

As opposed to (8), action (9) gives, upon variation with respect to $\omega_{a}^{\prime}{ }^{I J}$,

$$
\begin{aligned}
\omega_{a}^{\prime I J} & =\omega_{a}^{I J}(e)+K_{a}{ }^{I J}, \\
K_{a}{ }^{I J} & =\frac{1}{2}\left(e_{a}^{I} e^{b J}-e_{a}^{J} e^{b I}\right) \frac{1}{\Omega^{2}} \partial_{b} \Omega^{2},
\end{aligned}
$$

where $\omega_{a}^{I J}(e)$ is the spin connection for pure gravity and $K_{a}{ }^{I J}$ is the contorsion supported by the matter field [9]. To construct a first order action equivalent to the second order

action in (8), it is necessary to add the term $-\frac{3}{4} \frac{1}{\Omega^{2}} e e_{I}^{a} e^{b I} \partial_{a} \Omega^{2} \partial_{b} \Omega^{2}$ to the action (9). The corresponding canonical analysis of the self-dual part of this modified action was studied in [10]. The conclusion in that paper, at the Hamiltonian level, is that the resulting constraints are non-polynomial in the phase space variables. In the present work, the self-dual part of the action (9) is studied. Our result is that polynomial constraints are obtained, as opposed to the case in $[10]$.

The system to be considered is described by the so called non-minimal self-dual EinsteinCartan-Klein-Gordon (ECKG) action 


$$
\begin{aligned}
S\left[e,{ }^{+4} A, \phi\right] & =\int d^{4} x e\left\{\Omega^{2+} \Sigma_{I J}^{a b}{ }^{+4} F_{a b}{ }^{I J}\left({ }^{+4} A\right)+\frac{\alpha}{2}\left(e_{I}^{a} e^{b I} \partial_{a} \phi \partial_{b} \phi+m^{2} \phi^{2}\right)\right\}, \\
\Sigma_{I J}^{a b} & :=\frac{1}{2}\left(e_{I}^{a} e_{J}^{b}-e_{J}^{a} e_{I}^{b}\right), \\
\Omega^{2} & :=1+\alpha \xi \phi^{2},
\end{aligned}
$$

where the parameter $\alpha$ is introduced only to allow a rescaling of the scalar field $\phi$. The value $\alpha=-1$ corresponds to the case usually found in the literature [8,10]. ${ }^{+4} F_{a b}{ }^{I J}\left({ }^{+4} A\right)$ is the curvature of the self-dual connection ${ }^{+4} A$.

Proceeding with the canonical analysis, the $3+1$ decomposition of space-time gives

$$
\begin{aligned}
S= & \int d t \int_{\Sigma} d^{3} x\left\{\underset{\sim}{\sim} \Omega^{2}\left[-\frac{1}{2} \epsilon^{i j k} \widetilde{E}_{i}^{a} \widetilde{E}_{j}^{b} F_{a b k}\right]+\left(-i \Omega^{2} \widetilde{E}_{i}^{a}\right) \mathcal{L}_{t} A_{a}^{i}\right\} \\
& +\int d t \int_{\Sigma} d^{3} x\left\{\left({ }^{+4} A \cdot t\right)^{i} \mathcal{D}_{a}\left(-i \Omega^{2} \widetilde{E}_{i}^{a}\right)+N^{b}\left(-i \Omega^{2} \widetilde{E}_{i}^{a}\right) F_{a b}{ }^{i}\right\} \\
& +\frac{\alpha}{2} \int d t \int_{\Sigma} d^{3} x\left\{N \widetilde{E}_{i}^{a} \widetilde{E}^{b i} \partial_{a} \phi \partial_{b} \phi-\frac{1}{\sim}\left[\mathcal{L}_{t} \phi-\mathcal{L}_{N} \phi\right]^{2}+\underset{\sim}{\sim}(\sigma)^{2} m^{2} \phi^{2}\right\} .
\end{aligned}
$$

In order to get (12) one performs the usual steps [1]. $\widetilde{E}_{i}^{a}$ is the densitizied inverse triad field, $A_{a}^{i}$ is the 3 -dimensional projection of the self-dual full connection (gravity +matter) and $F_{a b}{ }^{i}$ is the corresponding curvature given by $F_{a b}{ }^{i}=\partial_{a} A_{b}^{i}-\partial_{b} A_{a}^{i}+\epsilon^{i}{ }_{j k} A_{a}^{j} A_{b}^{k} .(\sigma)^{2}$ is the determinant of the 3-dimensional spatial metric $q_{a b}$, expressed as a function of $\widetilde{E}_{i}^{a}$ and $\epsilon^{i j k}$ is the volume element of the 3 -dimensional internal metric $q_{i j}=\operatorname{diag}(+1,+1,+1)$. Both sets of indices run from 1 to 3 .

From (12) one finds the momentum variables associated with the gravitational and scalar fields. They are given by $\tilde{\pi}_{i}^{a}$ and $\tilde{\pi}_{\phi}$, where

$$
\begin{aligned}
& \widetilde{\pi}_{i}^{a}:=-i \Omega^{2} \widetilde{E}_{i}^{a}, \\
& \tilde{\pi}_{\phi}:=\frac{\delta S}{\delta\left(\mathcal{L}_{t} \phi\right)}=-\frac{\alpha}{\sim}\left[\mathcal{L}_{t} \phi-\mathcal{L}_{N} \phi\right] .
\end{aligned}
$$

Then, plugging (13) into (12), one arrives at

$$
S=\int d t \int_{\Sigma} d^{3} x\left\{\widetilde{\pi}_{i}^{a} \mathcal{L}_{t} A_{a}^{i}+\widetilde{\pi}_{\phi} \mathcal{L}_{t} \phi-\left(N^{*} \widetilde{\widetilde{C}}+\left({ }^{+4} A \cdot t\right)^{i} \widetilde{\mathcal{G}}_{i}+N^{a} \widetilde{\mathcal{V}}_{a}\right)\right\}
$$

where 


$$
\begin{aligned}
& \widetilde{\widetilde{C}}:=-\frac{1}{2}\left(\Omega^{2}\right)^{2} \epsilon^{i j k} \widetilde{\pi}_{i}^{a} \widetilde{\pi}_{j}^{b} F_{a b k}+\frac{\alpha}{2} \Omega^{2} \widetilde{\pi}_{i}^{a} \widetilde{\pi}^{b i} \partial_{a} \phi \partial_{b} \phi-\frac{1}{2 \alpha}\left(\Omega^{2}\right)^{3}\left(\widetilde{\pi}_{\phi}\right)^{2}+\frac{\alpha}{2} i\left(\operatorname{det} \widetilde{\pi}_{i}^{a}\right) m^{2} \phi^{2}, \\
& \widetilde{\mathcal{G}}_{i}:=-\mathcal{D}_{a} \widetilde{\pi}_{i}^{a}, \\
& \widetilde{\mathcal{V}}_{a}:=-\widetilde{\pi}_{i}^{b} F_{b a}{ }^{i}+\widetilde{\pi}_{\phi} \partial_{a} \phi .
\end{aligned}
$$

are the scalar, vector and Gauss constraints, respectively. In (14), the Lagrange multiplier $\underset{\sim}{\sim}$ was redefined to $\underset{\sim}{\sim}=\frac{N}{\sim}$. Note that the set of constraints (15) is polynomial in the phase space variables $A_{a}^{i}, \tilde{\pi}_{i}^{a}, \phi$ and $\tilde{\pi}_{\phi}$, where the symplectic structure is given by

$$
\begin{gathered}
\left\{A_{a}^{i}(x), \tilde{\pi}_{j}^{b}(y)\right\}=\delta_{a}^{b} \delta_{j}^{i} \delta^{3}(x, \tilde{y}), \\
\left\{\phi(x), \tilde{\pi}_{\phi}(y)\right\}=\delta^{3}(x, \tilde{y}) .
\end{gathered}
$$

Let us compare the actions (四) and (14). We find the same number of constraints which, nevertheless, have extra terms containing the scalar field. Besides, the new momenta have contributions arising both from the scalar field and the gravity sector.

In order to count the number of degrees of freedom of the theory it is necessary to classify the constraints in terms of their algebra. We find it convenient to use the smeared form of the constraints on $\Sigma$. If $\underset{\sim}{N}, N^{a}$ and $v^{i}$ are arbitrary tensor fields, then one defines

$$
\begin{aligned}
& C(\stackrel{N}{\sim}):=\int_{\Sigma} d^{3} x \stackrel{N}{\sim} \widetilde{\widetilde{C}}(x) \\
&= \int_{\Sigma} d^{3} x \stackrel{N}{\sim}\left\{-\frac{1}{2}\left(\Omega^{2}\right)^{2} \epsilon^{i j k} \widetilde{\pi}_{i}^{a} \widetilde{\pi}_{j}^{b} F_{a b k}+\frac{\alpha}{2} \Omega^{2} \widetilde{\pi}_{i}^{a} \widetilde{\pi}^{b i} \partial_{a} \phi \partial_{b} \phi\right. \\
&\left.\quad-\frac{1}{2 \alpha}\left(\Omega^{2}\right)^{3}\left(\widetilde{\pi}_{\phi}\right)^{2}+\frac{\alpha}{2} i\left(\operatorname{det} \widetilde{\pi}_{i}^{a}\right) m^{2} \phi^{2}\right\} \\
& C(\vec{N}):= \int_{\Sigma} d^{3} x N^{a} \widetilde{\mathcal{V}}_{a}(x)=\int_{\Sigma} d^{3} x N^{a}\left\{-\widetilde{\pi}_{i}^{b} F_{b a}{ }^{i}+\widetilde{\pi}_{\phi} \partial_{a} \phi\right\} \\
& G(v):=\int_{\Sigma} d^{3} x v^{i} \widetilde{\mathcal{G}}_{i}(x)=\int_{\Sigma} d^{3} x v^{i}\left\{-\mathcal{D}_{a} \widetilde{\pi}_{i}^{a}\right\}
\end{aligned}
$$

A combination of the vector and Gauss constraints yields the so called diffeomorphisms constraint. In terms of the vector field $\vec{N}$, this constraint has the form

$$
D(\vec{N}):=\int_{\Sigma} d^{3} x N^{a}\left[\widetilde{\mathcal{V}}_{a}+A_{a}^{i} \widetilde{\mathcal{G}}_{i}\right](x)=\int_{\Sigma} d^{3} x N^{a}\left[-\widetilde{\pi}_{i}^{b} F_{b a}{ }^{i}+A_{a}^{i}\left(-\mathcal{D}_{b} \tilde{\pi}_{i}^{b}\right)\right]
$$

The following results are useful in dealing with the algebra of constraints 


$$
\begin{array}{rlrl}
\frac{\delta C(\underset{\sim}{N})}{\delta A_{c}^{l}} & =-\epsilon_{l}{ }^{i j} \mathcal{D}_{b}\left(\stackrel{N}{\sim}\left(\Omega^{2}\right)^{2} \widetilde{\pi}_{i}^{c} \widetilde{\pi}_{j}^{b}\right), & & \\
\frac{\delta C(\underset{\sim}{N})}{\delta \widetilde{\pi}_{l}^{c}} & =N\left[-\left(\Omega^{2}\right)^{2} \epsilon^{l j k} \widetilde{\pi}_{j}^{a} F_{c a k}+\alpha \Omega^{2} \widetilde{\pi}^{a l} \partial_{a} \phi \partial_{c} \phi+\left(\frac{\alpha}{2} i m^{2} \phi^{2}\right)\left(\frac{3}{3 !} \eta_{a b c} \widetilde{\pi}_{j}^{a} \widetilde{\pi}_{k}^{b} \epsilon^{j k l}\right),\right] \\
\frac{\delta G(v)}{\delta A_{c}^{l}} & =-v^{i} \epsilon_{i l}{ }^{k} \widetilde{\pi}_{k}^{c}, & & \frac{\delta G(v)}{\delta \widetilde{\pi}_{l}^{c}}=\mathcal{D}_{c} v^{l}, \\
\frac{\delta D(\vec{N})}{\delta A_{c}^{l}} & =-\mathcal{L}_{\vec{N}} \widetilde{\pi}_{l}^{c}, & \frac{\delta D(\vec{N})}{\delta \widetilde{\pi}_{l}^{c}}=+\mathcal{L}_{\vec{N}} A_{c}^{l} .
\end{array}
$$

Then, by using (19), the algebra of constraints turns out to be

$$
\begin{aligned}
& \{C(\underset{\sim}{\sim}), C(\underset{\sim}{\sim})\}=-D(\vec{K})+G\left(K^{a} A_{a}\right), \\
& \{C(\underset{N}{\sim}), D(\vec{M})\}=-C\left(\mathcal{L}_{\vec{M}} N\right), \\
& \{C(\stackrel{N}{\sim}), G(v)\}=0, \\
& \{D(\vec{N}), D(\vec{M})\}=D([\vec{N}, \vec{M}]), \\
& \{D(\vec{N}), G(v)\}=G\left(\mathcal{L}_{\vec{N}} v\right), \\
& \{G(w), G(v)\}=D(-[w, v]) .
\end{aligned}
$$

In (20), the vector field $\vec{K}$ is defined by $K^{a}:=\left(\Omega^{2}\right)^{4}\left(\widetilde{\pi}_{i}^{a} \widetilde{\pi}^{b i}\right)\left(N \partial_{b} M-M \partial_{b} N\right)$, while the commutator of internal vectors is $[w, v]^{i}:=\epsilon^{i}{ }_{j k} w^{j} v^{k}$. Also, the commutator of spatial vectors is defined by $[\vec{N}, \vec{M}]^{a}:=\mathcal{L}_{\vec{N}} M^{a}$, as usual.

The set of constraints (20) is first class. The counting of degrees of freedom leads to: $2(9)+2(1)-2(7)=6$ phase space variables per point on $\Sigma$, which implies 3 complex degrees

of freedom: two for the gravitational field and one for the scalar field. To recover the real sector of the theory, i.e. three real degrees of freedom per point, one has to supply additional constraints on the phase space variables $A_{a}^{i}, \widetilde{\pi}_{i}^{a}, \phi, \widetilde{\pi}_{\phi}$. This is the subject of the following section.

\section{REAL DEGREES OF FREEDOM}

The real sector of the theory is recovered by extending the corresponding steps developed for pure gravity in [5]. To begin with, let us consider the action (14) in the explicit form 


$$
S=\int d t \int_{\Sigma} d^{3} x\left\{\left(-i \Omega^{2} \widetilde{E}_{i}^{a}\right) \mathcal{L}_{t} A_{a}^{i}+\widetilde{\pi}_{\phi} \mathcal{L}_{t} \phi-\left(N^{*} \widetilde{\widetilde{C}}+\left({ }^{+4} A \cdot t\right)^{i} \widetilde{\mathcal{G}}_{i}+N^{a} \widetilde{\mathcal{V}}_{a}\right)\right\}
$$

where

$$
\begin{aligned}
\widetilde{\widetilde{C}}:= & \left(\Omega^{2}\right)^{2}\left(-\frac{1}{2} \epsilon^{i j k}\right)\left(-i \Omega^{2} \widetilde{E}_{i}^{a}\right)\left(-i \Omega^{2} \widetilde{E}_{j}^{b}\right) F_{a b k}+\frac{\alpha}{2} \Omega^{2}\left(-i \Omega^{2} \widetilde{E}_{i}^{a}\right)\left(-i \Omega^{2} \widetilde{E}^{b i}\right) \partial_{a} \phi \partial_{b} \phi \\
& -\frac{1}{2 \alpha}\left(\Omega^{2}\right)^{3}\left(\widetilde{\pi}_{\phi}\right)^{2}+\frac{\alpha}{2} i\left(-i \Omega^{2}\right)^{3}\left(\operatorname{det} \widetilde{E}_{i}^{a}\right) m^{2} \phi^{2}, \\
\widetilde{\mathcal{G}}_{i}:= & -\mathcal{D}_{a}\left(-i \Omega^{2} \widetilde{E}_{i}^{a}\right) \\
\widetilde{\mathcal{V}}_{a}:= & -\left(-i \Omega^{2} \widetilde{E}_{i}^{b}\right) F_{b a}{ }^{i}+\widetilde{\pi}_{\phi} \partial_{a} \phi .
\end{aligned}
$$

Step (i) in the construction consists of splitting each one of the fields involved in (22) into their real and imaginary parts,

$$
\widetilde{E}^{a i}=\widetilde{e}^{a i}+i \widetilde{\mathcal{E}}^{a i} \quad, \quad A_{a i}=M_{a i}+i V_{a i} \quad, \phi=\phi_{1}+i \phi_{2} \quad, \widetilde{\pi}_{\phi}=\widetilde{\pi}_{1}+i \widetilde{\pi}_{2} .
$$

Now the key point, implemented as part of step (ii), is to promote each one of the real and imaginary parts to independent variables, which implies that the enlarged phase space has $2[4 \times 9+4]=80$ degrees of freedom per point on $\Sigma$. Next, one has to determine the corresponding momenta, which results in the following constraints,

$$
\begin{gathered}
\Phi_{\mathcal{E} a i}:=\Pi_{\mathcal{E} a i} \quad, \quad \Phi_{e a i}:=\Pi_{e a i} \quad, \quad \Phi_{M}^{a i}:=\Pi_{M}^{a i}+i \Omega_{1}^{2} \widetilde{e}^{a i} \quad, \quad \Phi_{V}^{a i}:=\Pi_{V}^{a i}-\Omega_{1}^{2} \widetilde{e}^{a i} \\
\Phi_{\pi_{1}}:=\Pi_{\pi_{1}} \quad, \quad \Phi_{\pi_{2}}:=\Pi_{\pi_{2}} \quad, \quad \Phi_{\phi_{1}}:=\Pi_{\phi_{1}}-\widetilde{\pi}_{1} \quad, \quad \Phi_{\phi_{2}}:=\Pi_{\phi_{2}}-i \widetilde{\pi}_{1}
\end{gathered}
$$

denoted generically by $\Pi$. Note that $\Pi_{M}^{a i}$ and $\Pi_{\phi_{2}}$ are purely imaginary, i.e. there are 40 primary constraints arising from the definition of momenta. The corresponding symplectic structure is

$$
\begin{array}{rrrr}
\left\{\widetilde{\mathcal{E}}^{a i}(x), \Pi_{\mathcal{E} b j}(y)\right\}:=\delta_{b}^{a} \delta_{j}^{i} \delta^{3}(\tilde{x}, y) & , & \left\{\widetilde{e}^{a i}(x), \Pi_{e b j}(y)\right\}:=\delta_{b}^{a} \delta_{j}^{i} \delta^{3}(\tilde{x}, y) \\
\left\{M_{a i}(x), \Pi_{M}{ }^{b j}(y)\right\}:=\delta_{a}^{b} \delta_{i}^{j} \delta^{3}(x, \tilde{y}) & , & \left\{V_{a i}(x), \Pi_{V}{ }^{b j}(y)\right\}:=\delta_{a}^{b} \delta_{i}^{j} \delta^{3}(x, \tilde{y}) \\
\left\{\phi_{1}(x), \Pi_{\phi_{1}}(y)\right\}:=\delta^{3}(x, \tilde{y}), & & \left\{\phi_{2}(x), \Pi_{\phi_{2}}(y)\right\}:=\delta^{3}(x, \tilde{y}) \\
\left\{\widetilde{\pi}_{1}(x), \Pi_{\pi_{1}}(y)\right\}:=\delta^{3}(\tilde{x}, y), & & \left\{\widetilde{\pi}_{2}(x), \Pi_{\pi_{2}}(y)\right\}:=\delta^{3}(\tilde{x}, y)
\end{array}
$$


As for step (iii), the reality conditions are chosen here as a generalization of (田), i.e.

$$
\begin{aligned}
\Psi_{\mathcal{E}}{ }^{a i} & :=\widetilde{\mathcal{E}}^{a i} \quad, \quad \Psi_{M a i}:=M_{a i}-\Gamma_{a i}(\widetilde{e})+\frac{1}{2} \epsilon_{i j}{ }^{k} e_{g}{ }^{j} \widetilde{e}^{c}{ }_{k} \frac{1}{\Omega_{1}^{2}} \partial_{c} \Omega_{1}^{2}, \\
\Psi_{\phi_{2}} & :=\phi_{2} \quad, \quad \Psi_{\pi_{2}}:=\widetilde{\pi}_{2} \quad,
\end{aligned}
$$

where $\Gamma_{a i}(e)$ is the 3 -dimensional spin connection. Again, $\Psi_{\mathcal{E}}^{a i}$ plays the role of enforcing $\widetilde{E}^{a i}$ to be real and $\Psi_{M a i}$ keeps $\widetilde{E}^{a i}$ real upon evolution. The term $\frac{1}{2} \epsilon_{i j}{ }^{k} e_{a}^{j} \widetilde{e}_{k}^{c} \frac{1}{\Omega_{1}^{2}} \partial_{c} \Omega_{1}^{2}$ is the real contribution of matter to the full connection $A_{a}^{i}$ : it is determined as the real term of the matter contribution to the spatial part of ${ }^{+} A$, upon variation of (11). Also, $\Psi_{\phi_{2}}, \Psi_{\pi_{2}}$ constrain the scalar field, $\phi$, to the real sector. At this stage there are 60 primary constraints, 20 of which arise from the reality conditions (26).

In the next step (iv), it is necessary to preserve upon evolution the full set of primary constraints (24) and (26). Before doing so, it is convenient to redefine some of them, $\Phi_{\text {eai }}$, $\Phi_{\phi_{1}}$ and $\Phi_{\pi_{1}}$, as

$$
\begin{aligned}
\Phi_{e a i}^{\prime}(x) & :=\Phi_{e a i}(x)+\lambda_{a i b j}(x, z) \Phi_{M}{ }^{b j}(z)+\varepsilon_{a i}{ }^{b j}(x, z) \Psi_{M b j}(z)+\vartheta_{a i b j}(x, z) \Phi_{V}{ }^{b j}(z), \\
\Phi_{\pi_{1}}^{\prime} & :=\Phi_{\pi_{1}}-i \Psi_{\phi_{2}}, \\
\Phi_{\phi_{1}}^{\prime}(x) & :=\Phi_{\phi_{1}}(x)+\mathcal{A}_{a i}(x, z) \Phi_{M}{ }^{a i}(z)+\mathcal{B}^{a i}(x, z) \Psi_{M a i}(z)+\mathcal{C}_{a i}(x, z) \Phi_{V}{ }^{a i}(z),
\end{aligned}
$$

where

$$
\begin{aligned}
\lambda_{a i b j}(x, y) & =-\frac{\delta \Psi_{M b j}(y)}{\delta \tilde{e}^{a i}(x)} \quad, \quad \varepsilon_{a i}{ }^{b j}(x, y)=i \Omega_{1}^{2}(y) \delta_{a}^{b} \delta_{i}^{j} \delta^{3}(x, \tilde{y}), \\
\vartheta_{a i b j}(x, y) & =-i \frac{\delta \Psi_{M b j}(y)}{\delta \tilde{e}^{a i}(x)} \quad, \\
\mathcal{A}_{a i}(x, y) & =-\frac{\delta \Psi_{M a i}(y)}{\delta \phi_{1}(x)} \quad, \quad \mathcal{B}^{a i}(x, y)=i \frac{\delta \Omega_{1}^{2}(y)}{\delta \phi_{1}(x)} \widetilde{e}^{a i}(y), \\
\mathcal{C}_{a i}(x, y) & =-i \frac{\delta \Psi_{M a i}(y)}{\delta \phi_{1}(x)} .
\end{aligned}
$$

Here, the Einstein summation convention for dummy indices is extended to the continuous case in such a way that it includes an implicit 3-dimensional integral for the repeated $z$ variable. Thus, for instance, the term $\lambda_{a i b j}(x, z) \Phi_{M}{ }^{b j}(z)$ means $\int_{\Sigma} d^{3} z \lambda_{a i b j}(x, z) \Phi_{M}{ }^{b j}(z)$. 
As the first step in classifying the constraints into first and second class one computes the Poisson brackets of the constraints $\Phi_{\mathcal{E}_{a i}}(x), \Psi_{\mathcal{E}}^{a i}(x), \Phi_{M}^{a i}(x), \Psi_{M a i}(x), \Phi_{\phi_{2}}(x), \Psi_{\phi_{2}}(x)$, $\Phi_{\pi_{2}}(x), \Psi_{\pi_{2}}(x), \Phi_{\phi_{1}}^{\prime}(x), \Phi_{V}^{a i}(x), \Phi_{e a i}^{\prime}(x)$ and $\Phi_{\pi_{1}}^{\prime}(x)$. In the standard notation, the result can be expressed as

$$
D=\left(\begin{array}{ccc}
A & 0 & 0 \\
0 & B & 0 \\
0 & 0 & C
\end{array}\right)
$$

where

$$
\begin{aligned}
& \Phi_{\mathcal{E}_{b j}}(y) \quad \Psi_{\mathcal{E}}^{b j}(y) \quad \Phi_{M}^{b j}(y) \quad \Psi_{M b j}(y) \\
& \Phi_{\mathcal{E}_{a i}(x)}(x) \quad-\delta_{a}^{b} \delta_{i}^{j} \delta^{3}(x, \tilde{y}) \quad 0 \quad 0 \\
& A=\Psi_{\mathcal{E}}^{a i}(x) \quad \delta_{b}^{a} \delta_{j}^{i} \delta^{3}(\tilde{x}, y) \quad 0 \quad 0 \quad 0 \quad \text {, } \\
& \begin{array}{ccccc}
\Phi_{M}^{a i}(x) & 0 & 0 & 0 & -\delta_{b}^{a} \delta_{j}^{i} \delta^{3}(\tilde{x}, y)
\end{array} \\
& \Psi_{M a i}(x) \quad 0 \quad 0 \quad \delta_{a}^{b} \delta_{i}^{j} \delta^{3}(x, \tilde{y}) \quad 0 \\
& \Phi_{\phi_{2}}(y) \quad \Psi_{\phi_{2}}(y) \quad \Phi_{\pi_{2}}(y) \quad \Psi_{\pi_{2}}(y) \\
& \Phi_{\phi_{2}}(x) \quad 0 \quad-\delta^{3}(\tilde{x}, y) \quad 0 \quad 0 \\
& B=\Psi_{\phi_{2}}(x) \delta^{3}(x, \tilde{y}) \quad 0 \quad 0 \quad 0 \quad \text {, } \\
& \Phi_{\pi_{2}}(x) \quad 0 \quad 0 \quad 0 \quad-\delta^{3}(x, \tilde{y}) \\
& \Psi_{\pi_{2}}(x) \quad 0 \quad 0 \quad \delta^{3}(\tilde{x}, y) \quad 0 \\
& \Phi_{\phi_{1}}^{\prime}(y) \quad \Phi_{V}^{b j}(y) \quad \Phi_{e b j}^{\prime}(y) \quad \Phi_{\pi_{1}}^{\prime}(y) \\
& \begin{array}{lllll}
\Phi_{\phi_{1}}^{\prime}(x) & 0 & \frac{\delta \Omega_{1}^{2}(y)}{\delta \phi_{1}(x)} \widetilde{e}^{b j}(y) & 0 & -\delta^{3}(\tilde{x}, y)
\end{array} \\
& C=\Phi_{V}{ }^{a i}(x)-\frac{\delta \Omega_{1}^{2}(x)}{\delta \phi_{1}(y)} \widetilde{e}^{a i}(x) \quad 0 \quad-\Omega_{1}^{2}(x) \delta_{b}^{a} \delta_{j}^{i} \delta^{3}(\tilde{x}, y) \quad 0 \quad \text {. } \\
& \Phi_{\text {eai }}^{\prime}(x) \quad 0 \quad \Omega_{1}^{2}(y) \delta_{a}^{b} \delta_{i}^{j} \delta^{3}(x, \tilde{y}) \quad 0 \quad 0 \\
& \begin{array}{lllll}
\Phi_{\pi_{1}}^{\prime}(x) & \delta^{3}(x, \tilde{y}) & 0 & 0 & 0
\end{array}
\end{aligned}
$$

From the structure of the matrices $(29 \sqrt{32})$ one concludes that the set of constraints arising from the definition of the momenta, together with those which come from the reality 
conditions are second class. On the other hand, the original set of constraints $\widetilde{\widetilde{C}}, \widetilde{\mathcal{G}}_{i}, \widetilde{\mathcal{V}}_{a}$, in the complex theory, were the generators of the gauge symmetry of the system. To show that this property remains so in the present real theory, one begins by redefining them in such a way that they have zero Poisson brackets with the second class constraints. Then, it should be verified that this redefinition preserves the first class character of the algebra among them. Recall that $\widetilde{\widetilde{C}}, \widetilde{\mathcal{G}}_{i}$ and $\widetilde{\mathcal{V}}_{a}$ depend only upon configuration variables (See (15) and (23)). Denoting any of them by $\mathcal{R}$, we find that the appropriate redefinition is

$$
\begin{aligned}
\mathcal{R}^{\prime}(x):= & \mathcal{R}(x)+I^{b j}(x, z) \Psi_{M b j}(z)+N_{b j}(x, z) \Phi_{V}^{b j}(z)+O^{b j}(x, z) \Phi_{e b j}^{\prime}(z)+ \\
& +H_{b j}(x, z) \Psi_{\mathcal{E}}{ }^{b j}(z)+P(x, z) \Psi_{\pi_{2}}(z)+Q(x, z) \Psi_{\phi_{2}}(z) \\
& +R(x, z) \Phi_{\phi_{1}}^{\prime}(z)+S(x, z) \Phi_{\pi_{1}}^{\prime}(z),
\end{aligned}
$$

where

$$
\begin{aligned}
I^{a i}(x, y) & =\left\{\Phi_{M}^{a i}(y), \mathcal{R}(x)\right\} \quad, \quad H_{a i}(x, y)=\left\{\Phi_{\mathcal{E}_{a i}}(y), \mathcal{R}(x)\right\} \\
N_{a i}(x, y) & =-\frac{1}{\Omega_{1}^{2}(y)}\left\{\Phi_{e a i}^{\prime}(y), \mathcal{R}(x)\right\} \quad, \quad R(x, y)=-\left\{\Phi_{\pi_{1}}^{\prime}(y), \mathcal{R}(x)\right\} \\
O^{a i}(x, y) & =\frac{1}{\Omega_{1}^{2}(y)}\left[\left\{\Phi_{V}^{a i}(y), \mathcal{R}(x)\right\}+\frac{\delta \Omega_{1}^{2}(y)}{\delta \phi_{1}(z)} \widetilde{e}^{a i}(y)\left\{\Phi_{\pi_{1}}^{\prime}(z), \mathcal{R}(x)\right\}\right] \\
P(x, y) & =\left\{\Phi_{\pi_{2}}(y), \mathcal{R}(x)\right\} \quad, \quad Q(x, y)=\left\{\Phi_{\phi_{2}}(y), \mathcal{R}(x)\right\} \\
S(x, y) & =\left\{\Phi_{\phi_{1}}^{\prime}(y), \mathcal{R}(x)\right\}-\frac{1}{\Omega_{1}^{2}(z)} \frac{\delta \Omega_{1}^{2}(z)}{\delta \phi_{1}(y)} \widetilde{e}^{b j}(z)\left\{\Phi_{e b j}^{\prime}(z), \mathcal{R}(x)\right\}
\end{aligned}
$$

The Poisson brackets between any pair $\mathcal{Q}^{\prime}$ and $\mathcal{R}^{\prime}$ is weakly zero. This can be shown by calculating

$$
\begin{aligned}
\left\{\mathcal{Q}^{\prime}(x), \mathcal{R}^{\prime}(y)\right\} \approx & \left\{\mathcal{Q}^{\prime}(x), \mathcal{R}(y)\right\}= \\
& -\frac{1}{\Omega_{1}^{2}(z)}\left\{\Phi_{\text {eai }}^{\prime}(z), \mathcal{Q}(x)\right\}\left\{\Phi_{V}^{a i}(z), \mathcal{R}(y)\right\} \\
& +\frac{1}{\Omega_{1}^{2}(z)}\left\{\Phi_{V}^{a i}(z), \mathcal{Q}(x)\right\}\left\{\Phi_{\text {eai }}^{\prime}(z), \mathcal{R}(y)\right\} \\
& -\left\{\Phi_{\pi_{1}}^{\prime}(z), \mathcal{Q}(x)\right\}\left\{\Phi_{\phi_{1}}^{\prime}(z), \mathcal{R}(y)\right\} \\
& +\left\{\Phi_{\phi_{1}}^{\prime}(z), \mathcal{Q}(x)\right\}\left\{\Phi_{\pi_{1}}^{\prime}(z), \mathcal{R}(y)\right\}
\end{aligned}
$$




$$
\begin{aligned}
& +\frac{1}{\Omega_{1}^{2}(z)} \frac{\delta \Omega_{1}^{2}(z)}{\delta \phi_{1}(\omega)} \widetilde{e}^{a i}(z)\left\{\Phi_{\pi_{1}}^{\prime}(\omega), \mathcal{Q}(x)\right\}\left\{\Phi_{\text {eai }}^{\prime}(z), \mathcal{R}(y)\right\} \\
& -\frac{1}{\Omega_{1}^{2}(\omega)} \frac{\delta \Omega_{1}^{2}(\omega)}{\delta \phi_{1}(z)} \widetilde{e}^{a i}(\omega)\left\{\Phi_{\text {eai }}^{\prime}(\omega), \mathcal{Q}(x)\right\}\left\{\Phi_{\pi_{1}}^{\prime}(z), \mathcal{R}(y)\right\}
\end{aligned}
$$

The above result was obtained by substituting (33) together with the fact that $\mathcal{Q}(x)$, $\mathcal{R}(y), \Psi_{M}, \Psi_{\mathcal{E}}, \Psi_{\pi_{2}}$ and $\Psi_{\phi_{2}}$ are independent of the momenta. Now, by using the explicit form of $\Phi_{e a i}^{\prime}, \Phi_{\phi_{1}}^{\prime}$ and $\Phi_{\pi_{1}}^{\prime}$ one finds, after a long (but otherwise direct) calculation

$$
\begin{aligned}
\left\{\mathcal{Q}^{\prime}(x), \mathcal{R}^{\prime}(y)\right\} \approx & -\frac{1}{\Omega_{1}^{2}(z)}\left\{\Phi_{\text {eai }}(z), \mathcal{Q}(x)\right\}\left\{\Phi_{V}{ }^{a i}(z), \mathcal{R}(y)\right\} \\
& +\frac{1}{\Omega_{1}^{2}(z)}\left\{\Phi_{V}{ }^{a i}(z), \mathcal{Q}(x)\right\}\left\{\Phi_{\text {eai }}(z), \mathcal{R}(y)\right\} \\
& -\left\{\Phi_{\pi_{1}}(z), \mathcal{Q}(x)\right\}\left\{\Phi_{\phi_{1}}(z), \mathcal{R}(y)\right\} \\
& +\left\{\Phi_{\phi_{1}}(z), \mathcal{Q}(x)\right\}\left\{\Phi_{\pi_{1}}(z), \mathcal{R}(y)\right\} \\
& +\frac{1}{\Omega_{1}^{2}(z)} \frac{\delta \Omega_{1}^{2}(z)}{\delta \phi_{1}(\omega)} \widetilde{e}^{a i}(z)\left\{\Phi_{\pi_{1}}(\omega), \mathcal{Q}(x)\right\}\left\{\Phi_{\text {eai }}(z), \mathcal{R}(y)\right\} \\
& -\frac{1}{\Omega_{1}^{2}(\omega)} \frac{\delta \Omega_{1}^{2}(\omega)}{\delta \phi_{1}(z)} \widetilde{e}^{a i}(\omega)\left\{\Phi_{\text {eai }}(\omega), \mathcal{Q}(x)\right\}\left\{\Phi_{\pi_{1}}(z), \mathcal{R}(y)\right\}
\end{aligned}
$$

The above expression is most suitably calculated in terms of the original complex phase space variables. The symplectic structure (16) yields

$$
\begin{gathered}
\left\{A_{a}^{i}(x), \widetilde{E}_{j}^{b}(y)\right\}=\left\{A_{a}{ }^{i}(x), \frac{i \widetilde{\pi}^{b}{ }_{j}(y)}{\Omega^{2}(y)}\right\}=\frac{i}{\Omega^{2}(y)} \delta_{a}^{b} \delta_{j}^{i} \delta^{3}(x, \tilde{y}), \\
\left\{\widetilde{E}_{i}^{a}(x), \widetilde{\pi}_{\phi}(y)\right\}=\left\{\frac{i \widetilde{\pi}^{a}(x)}{\Omega^{2}(x)}, \widetilde{\pi}_{\phi}(y)\right\}=-\frac{1}{\Omega^{2}(x)} \widetilde{E}_{i}^{a}(x) \frac{\delta \Omega^{2}(x)}{\delta \phi(y)} .
\end{gathered}
$$

Upon substitution of these expressions in (36) one obtains

$$
\begin{aligned}
\left\{\mathcal{Q}^{\prime}(x), \mathcal{R}^{\prime}(y)\right\} \approx & \left\{A_{a}^{i}(z), \widetilde{E}_{j}^{b}(\omega)\right\}\left(\frac{\delta \mathcal{Q}(x)}{\delta A_{a}^{i}(z)} \frac{\delta \mathcal{R}(y)}{\delta \widetilde{E}_{i}^{b}(z)}-\frac{\delta \mathcal{Q}(x)}{\delta \widetilde{E}_{j}^{b}(z)} \frac{\delta \mathcal{R}(y)}{\delta A_{a}^{i}(z)}\right) \\
& +\left\{\phi(z), \widetilde{\pi}_{\phi}(\omega)\right\}\left(\frac{\delta \mathcal{Q}(x)}{\delta \phi(z)} \frac{\delta \mathcal{R}(y)}{\delta \widetilde{\pi}_{\phi}(z)}-\frac{\delta \mathcal{Q}(x)}{\delta \widetilde{\pi}_{\phi}(z)} \frac{\delta \mathcal{R}(y)}{\delta \phi(z)}\right) \\
& +\left\{\widetilde{E}_{i}^{a}(z), \widetilde{\pi}_{\phi}(\omega)\right\}\left(\frac{\delta \mathcal{Q}(x)}{\delta \widetilde{E}_{i}^{a}(z)} \frac{\delta \mathcal{R}(y)}{\delta \widetilde{\pi}_{\phi}(\omega)}-\frac{\delta \mathcal{Q}(x)}{\delta \widetilde{\pi}_{\phi}(\omega)} \frac{\delta \mathcal{R}(y)}{\delta \widetilde{E}_{i}^{a}(z)}\right) \\
\approx & \{\mathcal{Q}(x), \mathcal{R}(y)\}(A, \pi),\left(\phi, \pi_{\phi}\right)
\end{aligned}
$$


In the last line, the Poisson brackets are taken with respect to the original complex symplectic structure (16). Therefore, it has been shown that the Poisson brackets between any pair of constraints $\widetilde{\widetilde{C}^{\prime}}, \widetilde{\mathcal{V}^{\prime}}, \widetilde{\mathcal{G}^{\prime}}$ are weakly zero.

Thus, the system is described by the following set of primary constraints: $\Phi_{\mathcal{E}_{a i}}(x)$, $\Psi_{\mathcal{E}}^{a i}(x), \Phi_{M}^{a i}(x), \Psi_{M a i}(x), \Phi_{\phi_{2}}(x), \Psi_{\phi_{2}}(x), \Phi_{\pi_{2}}(x), \Psi_{\pi_{2}}(x), \Phi_{\phi_{1}}^{\prime}(x), \Phi_{V}^{a i}(x), \Phi_{\text {eai }}^{\prime}(x)$, $\Phi_{\pi_{1}}^{\prime}(x), \widetilde{\widetilde{C}^{\prime}}, \widetilde{\mathcal{V}^{\prime}}$ and $\widetilde{\mathcal{G}^{\prime}}$. Now, following the Dirac method, the time conservation of the constraints is imposed using the Hamiltonian density

$$
\begin{aligned}
\mathcal{H}= & \mu^{\mathcal{E}}{ }^{a i} \Phi_{\mathcal{E}_{a i}}+\mu^{\mathcal{E}}{ }_{a i} \Psi_{\mathcal{E}}{ }^{a i}+\mu^{M i}{ }_{a i} \Phi_{M}{ }^{a i}+\mu^{M a i} \Psi_{M a i}+\mu^{\phi_{2}} \Phi_{\phi_{2}}+\nu^{\phi_{2}} \Psi_{\phi_{2}}+ \\
& +\mu^{\pi_{2}} \Phi_{\pi_{2}}+\nu^{\pi_{2}} \Psi_{\pi_{2}}+\mu^{\phi_{1}} \Phi_{\phi_{1}}^{\prime}+\mu^{V}{ }_{a i} \Phi_{V}{ }^{a i}+\mu^{e a i} \Phi_{e a i}^{\prime}++\mu^{\pi_{1}} \Phi_{\pi_{1}}^{\prime}+ \\
& +N \widetilde{\widetilde{C}^{\prime}}+N^{a} \widetilde{\mathcal{V}_{a}^{\prime}}+N^{i} \widetilde{\mathcal{G}_{i}^{\prime}},
\end{aligned}
$$

where no 3-dimensional integral is involved.

From Eqs. (33) and (39) one finds that the Poisson brackets between $\widetilde{\widetilde{C}^{\prime}}, \widetilde{\mathcal{V}^{\prime}}, \widetilde{\mathcal{G}^{\prime}}$ and $H=\int_{\Sigma} d^{3} x \mathcal{H}(x)$ are weakly zero. Finally, since the set of constraints $\Phi_{\mathcal{E}_{a i}}(x), \Psi_{\mathcal{E}}{ }^{a i}(x)$, $\Phi_{M}^{a i}(x), \Psi_{M a i}(x), \Phi_{\phi_{2}}(x), \Psi_{\phi_{2}}(x), \Phi_{\pi_{2}}(x), \Psi_{\pi_{2}}(x), \Phi_{\phi_{1}}^{\prime}(x), \Phi_{V}^{a i}(x), \Phi_{e a i}^{\prime}(x)$ and $\Phi_{\pi_{1}}^{\prime}(x)$ is second class, the Lagrange multipliers $\mu^{\mathcal{E}^{a i}}, \mu^{\mathcal{E}}{ }_{a i}, \mu^{M}{ }_{a i}, \mu^{M^{a i}}, \mu^{\phi_{2}}, \nu^{\phi_{2}}, \mu^{\pi_{2}}, \nu^{\pi_{2}}, \mu^{\phi_{1}}, \mu^{V}{ }_{a i}$, $\mu^{e a i}, \mu^{\pi_{1}}$ are determined, and shown to be zero. In other words, there are no secondary constraints and the total Hamiltonian density is given by

$$
\mathcal{H}_{\text {Total }}=\underset{\sim}{\sim} \widetilde{\widetilde{C}^{\prime}}+N^{a}{\widetilde{\mathcal{V}^{\prime}}}_{a}+N^{i} \widetilde{\mathcal{G}}^{\prime}{ }_{i}
$$

which is a combination of the first class constraints only.

Now, let us count the physical degrees of freedom in terms of the real variables that we have introduced. Recall that the enlarged phase space, with configuration variables (23), has dimension $2 \times 9 \times 4+2 \times 4=80$ per space point. Since there are $6 \times 9+6 \times 1=60$ second class constraints (24) and (26), the partially reduced phase space has dimension 20 . The corresponding partially reduced symplectic structure can be obtained by using Dirac brackets. To this end, the inverse of the second class constraints Poisson brackets matrix (29) is needed. Its calculation produces 


$$
D^{-1}=\left(\begin{array}{ccc}
A^{-1} & 0 & 0 \\
0 & B^{-1} & 0 \\
0 & 0 & C^{-1}
\end{array}\right)
$$

where

$$
\begin{gathered}
A^{-1}=\left(\begin{array}{cccc}
0 & \delta_{b}^{a} \delta_{j}^{i} \delta^{3}(\tilde{x}, y) & 0 & 0 \\
-\delta_{a}^{b} \delta_{i}^{j} \delta^{3}(x, \tilde{y}) & 0 & 0 & 0 \\
0 & 0 & 0 & \delta_{a}^{b} \delta_{i}^{j} \delta^{3}(x, \tilde{y}) \\
0 & 0 & -\delta_{b}^{a} \delta_{j}^{i} \delta^{3}(\tilde{x}, y) & 0
\end{array}\right), \\
C^{-1}=\left(\begin{array}{cccc}
0 & \delta^{3}(x, \tilde{y}) & 0 & 0 \\
-\delta^{3}(\tilde{x}, y) & 0 & 0 & 0 \\
0 & 0 & 0 & \delta^{3}(\tilde{x}, y) \\
0 & 0 & -\delta^{3}(x, \tilde{y}) & 0
\end{array}\right), \\
0 \quad \begin{array}{ccc}
0 \\
0
\end{array} \\
0 \\
0
\end{gathered}
$$

The use of (4245), leads to

$$
\begin{aligned}
\{f(x), g(y)\}^{*}= & +\{f(x), g(y)\} \\
& -\left\{f(x), \Phi_{\mathcal{E}_{a i}}(z)\right\}\left\{\Psi_{\mathcal{E}}^{a i}(z), g(y)\right\}+\left\{f(x), \Psi_{\mathcal{E}}^{a i}(z)\right\}\left\{\Phi_{\mathcal{E}_{a i}}(z), g(y)\right\} \\
& -\left\{f(x), \Phi_{M}{ }^{a i}(z)\right\}\left\{\Psi_{M a i}(z), g(y)\right\}+\left\{f(x), \Psi_{M a i}(z)\right\}\left\{\Phi_{M}{ }^{a i}(z), g(y)\right\} \\
& -\left\{f(x), \Phi_{\phi_{2}}(z)\right\}\left\{\Psi_{\phi_{2}}(z), g(y)\right\}+\left\{f(x), \Psi_{\phi_{2}}(z)\right\}\left\{\Phi_{\phi_{2}}(z), g(y)\right\} \\
& -\left\{f(x), \Phi_{\pi_{2}}(z)\right\}\left\{\Psi_{\pi_{2}}(z), g(y)\right\}+\left\{f(x), \Psi_{\pi_{2}}(z)\right\}\left\{\Phi_{\pi_{2}}(z), g(y)\right\} \\
& -\left\{f(x), \Phi_{\phi_{1}}^{\prime}(z)\right\}\left\{\Phi_{\pi_{1}}^{\prime}(z), g(y)\right\}+\left\{f(x), \Phi_{\pi_{1}}^{\prime}(z)\right\}\left\{\Phi_{\phi_{1}}^{\prime}(z), g(y)\right\} \\
& -\left\{f(x), \Phi_{V}{ }^{a i}(z)\right\} \frac{1}{\Omega_{1}^{2}(z)}\left\{\Phi_{e a i}^{\prime}(z), g(y)\right\}
\end{aligned}
$$




$$
\begin{aligned}
& +\left\{f(x), \Phi_{\text {eai }}^{\prime}(z)\right\} \frac{1}{\Omega_{1}^{2}(z)}\left\{\Phi_{V}^{a i}(z), g(y)\right\} \\
& -\left\{f(x), \Phi_{\pi_{1}}^{\prime}(z)\right\}\left[\frac{1}{\Omega_{1}^{2}(w)} \frac{\delta \Omega_{1}^{2}(\omega)}{\delta \phi_{1}(z)} e^{a i}(w)\right]\left\{\Phi_{\text {eai }}^{\prime}(w), g(y)\right\} \\
& +\left\{f(x), \Phi_{\text {eai }}^{\prime}(z)\right\}\left[\frac{1}{\Omega_{1}^{2}(z)} \frac{\delta \Omega_{1}^{2}(z)}{\delta \phi_{1}(\omega)} e^{a i}(z)\right]\left\{\Phi_{\pi_{1}}^{\prime}(\omega), g(y)\right\}
\end{aligned}
$$

for the Dirac brackets of any two functions $f$ and $g$ on the enlarged phase space. Finally, upon partial reduction, the canonical variables are $V_{a}^{i}, \pi_{j}^{b}=-\Omega_{1}^{2} e_{j}^{b}, \phi_{1}, \pi_{1}$ and the reduced symplectic structure is just

$$
\begin{aligned}
& \left\{V_{a}^{i}(x), \pi_{j}^{b}(y)\right\}^{*}=\delta_{a}^{b} \delta_{j}^{i} \delta^{3}(x, \tilde{y}), \\
& \left\{\phi_{1}(x), \pi_{1}(y)\right\}^{*}=\delta^{3}(x, \tilde{y}) .
\end{aligned}
$$

In the same way as in the pure gravity case [5], the first class constraints (15) turn out to be either purely real or purely imaginary in the above partially reduced phase space. Then, the physical phase space has dimension $20-2 \times 7=6$, as expected for a real scalar field coupled to real gravity.

\section{CONCLUSIONS AND PERSPECTIVES}

Previous results yielding the identification in phase space of the real sector of pure complex gravity [5] have been successfully extended in this work to incorporate the case of a scalar field non minimally coupled to gravity, starting from (complex) Ashtekar variables. This provides further support for the general validity of the method proposed.

The procedure is as follows: the complex canonical variables are splitted into real and imaginary parts, each of which is taken as an independent new configuration variable. The corresponding momenta are subsequently defined from the action, leading to primary constraints. The real sector of the theory is next defined by introducing appropriate reality conditions in the form of additional primary constraints. This is possible because the original phase space has been extended. The whole set of constraints is next classified into first and second class, after imposing the conservation of the primary constraints upon evolution. 
Finally, one faces the problem of how to conveniently deal with the resulting second class constraints, which include the reality conditions.

The advantages of our approach are: i) Reality conditions are incorporated as true second class constraints within the canonical description of an extended phase space, uniquely associated to each physical system. ii) It leads to the standard Dirac's method of counting the real physical degrees of freedom arising from an originally complex theory. iii) Although we start from a pair of reality conditions (四), only the first is truly an input, because the second condition appears as the consequence of demanding the conservation of the former upon evolution.

As opposed to [10], we have presented here a theory for a scalar field non minimally coupled to gravity, leading to polynomial constraints, using Ashtekar variables. Unfortunately, the non polynomiality shows up after implementing the reality conditions, in the process of identifying the real sector of the theory . This happens either for the non polynomial form of the reality conditions (26), or for their polynomial realization. Recently, however, certain non polynomial constraints have been shown to be tractable in the quantum theory [3,[1],[12]. Interestingly enough, in our case the whole non polynomiality is encoded in the single function $\Gamma_{a i}$ appearing in (26). It certainly remains an open problem to determine whether or not the results presented here may provide a tractable alternative to deal with the quantum situation.

The use of Dirac brackets, which is the standard way of eliminating the second class constraints, yields the expected real non polynomial form of the theory. For example, it leads to the Palatini canonical form in the case of pure gravity 国. To explore an alternative preventing the use of Dirac brackets in the pure gravity case, we have implemented the conversion of the full set of second class constraints into a first class set, following the method of [7]. Thus, we have rewritten pure real gravity as a theory involving an alternative set of first class constraints, which, for example, has not been previously done starting from the Palatini formulation with second class constraints. However, their physical meaning, together with their usefulness in a quantum theory still needs to be clarified. The method 
of [7] works whenever the Poisson brackets matrix of the original second class constraints is independent of the phase space variables. This is indeed the case for pure gravity, but not for the scalar field non minimally coupled to gravity considered in this work. Hence, the application of the same strategy to the latter theory would first require an extension of the method in Ref. [7].

\section{ACKNOWLEDGEMENTS}

Partial support is acknowledged from CONACyT grant 3141P-E9608 and UNAMDGAPA-IN100397. MM thanks all the members of the relativity group of the Department of Physics and Astronomy of the University of Pittsburgh for their warm hospitality. MM's postdoctoral fellowship is funded through the CONACyT of México, fellow number 91825. Also MM thanks support from the Sistema Nacional de Investigadores. 


\section{REFERENCES}

[1] C. Rovelli, living reviews in relativity http://www.livingreviews.org/Articles/Volume1/1998-1rovelli (1998).

C. Rovelli, Strings, loops and others: a critical survey of the present approaches to quantum gravity. Plenary lecture on quantum gravity at the GR15 conference, Pune, India. gr-qc archives No. 9803024.

[2] A. Ashtekar, C. Rovelli and L. Smolin, Phys. Rev. Lett. 69, 237 (1992).

C. Rovelli and L. Smolin, Nucl. Phys. B 442, 593 (1995).

C. Rovelli and L. Smolin, Erratum Nucl. Phys. B 456, 753 (1995).

A. Ashtekar and J. Lewandowski, Class. Quantum Grav. 14, A55 (1997).

[3] T. Thiemann, Phys. Lett. B 380, 257 (1996).

T. Thiemann, Class. Quantum Grav. 15, 839 (1998).

[4] A. Ashtekar, Phys. Rev. Lett. 77, 3288 (1986).

A. Ashtekar, Phys. Rev. D 36, 1587 (1987).

A. Ashtekar, Lectures on Non-Perturbative Canonical Gravity (Notes prepared in collaboration with $R$ S Tate) (World Scientific, Singapore, 1991).

[5] H.A. Morales-Técotl, L.F. Urrutia and J.D. Vergara, Class. Quantum Grav. 13, 2933 (1996).

[6] I.A. Batalin and I.V. Tyutin, Int. J. Mod. Phys. A 6, 3255 (1991).

[7] R. Amorim and A. Das, Mod. Phys. Lett. A 9, 3543 (1994).

[8] C. Kiefer, Phys. Lett. B 225, 227 (1989).

[9] F.W. Hehl, J.D. McCrea, E.W. Mielke and Y. Ne'eman, Phys. Rep. 258, 1 (1995).

[10] R. Capovilla, Phys. Rev. D 46, 1450 (1992).

[11] J.F. Barbero, Phys. Rev. D 51, 5507 (1995). 
[12] J.F. Barbero Phys. Rev. D 49, 6935 (1994).

J.F. Barbero Phys. Rev. D 51, 5498 (1995). 